\title{
Uptake of Medium-Size Gold Particles in the Nucleus of Living Cells
}

\author{
Ahmad Sohrabi Kashani ${ }^{1}$, Simona Badilescu ${ }^{1}$, Alisa Piekny ${ }^{2}$, Muthukumaran Packirisamy ${ }^{1}$ \\ ${ }^{1}$ Optical Bio-Microsystem Lab., Mechanical and Industrial Engineering, Concordia University \\ 1455 De Maisonneuve Blvd. W., H3G 1M8, Montreal, Quebec, Canada \\ Ahmad.sohrabi@encs.concordia.ca; simonabadilescu0@gmail.com; mpackir@encs.concordia.ca \\ ${ }^{2}$ Biology Department, Concordia University \\ 7141 Sherbrook Street W., H4B 1R6, Montreal, Quebec, Canada \\ Alisa.piekny@concordia.ca
}

\section{Extended Abstract}

The unique physical and structural properties of nanoparticles have made them an attractive nanomaterial for biomedical application such as intercellular drug delivery, cancer treatments, and medical imaging. Gold nanoparticles, in particular, have numerous advantages due to ease of conjugate functional biomolecules. They are stable, and have tuneable optical properties. The cellular uptake of nanoparticles play a significant role in their biomedical application, so it is essential to study their interaction with living cells before using them for possible biomedical applications [1-2]. The nucleus of cells is the most important part of cells, controlling their biological function such as growth, proliferation, and apoptosis. Therefore, targeting the cells' nucleus is the initial goal for drug delivery and cancer therapy which can help to improve the therapeutic efficiency of anti-cancer drugs [3]. It has been shown that physical and structural properties of NPs including size, shape and surface coating all may affect their interaction and the ability for cellular entry $[4,5,6]$.

In the present work, various imaging techniques were used to study the internalization ability of two different types of the gold particles; citrate-capped gold nanoparticles (AuNPs) and incinerated gold particles (IAuPs), to both healthy (HFF1) and cancerous cells (HeLa). Dynamic Light Scattering (DLS) and Scanning Electron Microscopy (SEM) imaging were used for physical characterization of particles. The characterization of particles showed that AuNPs are spherical and their sizes vary from 100 to $200 \mathrm{~nm}$ while IAuPs are larger and non-uniform in size and their average diameter is around 4500 $\mathrm{nm}$. Light microscopy and SEM were employed to investigate the localization of particles into the cells. The results showed that both particles are non-toxic to the HeLa and HFF-1 cell lines and no cell death was observed after a long incubation time. Moreover, it was observed that particles tend to accumulate at the different subcellular location, depending on their size. The results revealed that several AuPNs can enter and accumulate in cells, mostly near the nucleus while fewer IAuPs were able to enter cells and their distribution in cells, unlike to AuPNs, was in the random fashion (mostly in cytosol and vacuoles). Interestingly, it was observed that few gold nanoparticle even larger particle (IAuPs) can enter the nucleus of HeLa cells. It was observed that during nucleus division, particles in cytosol randomly are incorporated into the nucleus. Further study on different entry mechanisms of IAuPs to the cells revealed that IAuPs are only able to enter nucleus during nuclear reformation after cells division. The internalization ability and non-toxicity of relatively large IAuPs may be exploited for different biomedical application such as imaging, diagnosis, and treatment.

\section{References}

[1] T. Sun, Y. S. Zhang, B. Pang, D. C. Hyun, M. Yang, and, Y. Xia, "Engineered nanoparticles for drug delivery in cancer therapy," Angewandte Chemie International Edition, vol. 53, no. 46, pp. 12320-12364, 2014.

[2] D. Beaudet, S. Badilescu, K. Kuruvinashetti, A. S. Kashani, D. Jaunky, S. Ouellette, A. Piekny, and M. Packirisamy, "Comparative study on cellular entry of incinerated ancient gold particles (Swarna Bhasma) and chemically synthesized gold particles," Scientific Reports., vol. 7, no. 1, p. 10678, 2017.

[3] D. H. M Dam, J. H. Lee, P. N. Sisco, D. T. Co, M. Zhang, Wasielewski, M. R. and T. W. Odom, "Direct observation of nanoparticle-cancer cell nucleus interactions" ACS nano, vol. 6, no.4, pp. 3318-3326, 2012.

[4] X. Wang, X. Hu, J. Li, A. C. M. Russe, N. Kawazoe, Y. Yang, and G. Chen, "Influence of cell size on cellular uptake of gold nanoparticles," Biomaterials science, vol. 4, no. 6, pp. 970-978. 2016. 
[5] X. Xie, J. Liao, X. Shao,Q. Li, and Y. Lin, "The Effect of shape on Cellular Uptake of Gold Nanoparticles in the forms of Stars, Rods, and Triangles," Scientific Reports, vol. 7, no. 1, p. 3827, 2017.

[6] K. Kuruvinashetti, A. S. Kashani, S. Badilescu, D. Beaudet, A. Piekny, and M. Packirisamy, "Intracellular Localized Surface Plasmonic Sensing for Subcellular Diagnosis," Plasmonic, pp. 1-10, 2017. 